\title{
On Equivalence and Feedback Equivalence to Finitely Computable Sampled Models
}

\author{
P. Di Giamberardino, S. Monaco and D. Normand-Cyrot
}

\begin{abstract}
The paper deals with the finiteness of the series expansions representing the sampled model of a nonlinear plant after coordinates transformation and state feedback. Such a property makes the computation of the exact sampled model possible by finite calculations: "finite computability". Necessary and sufficient conditions are given for the class of controllable driftless dynamics. Some constructive approaches to the computation of the solutions are discussed. The results are extended to the class of underactuated mechanical systems.
\end{abstract}

\section{INTRODUCTION}

Since the early 90's a big effort was devoted to the study of nonlinear plants which are not linearizable under feedback. Several control problems were posed and solved for the subclass of nonholonomic systems motivated by their relevance in modeling real systems (see [5] and the 172 references therein).

In this context a new design approach was proposed in [7] for a class of models which includes feedback linearizable dynamics and several nonholonomic ones: it was suggested to make use of a preliminary static state feedback and a coordinates transformation for rendering solvable, in the discrete time domain, the equivalent control problem over the sampled model. An "hybrid" control scheme is thus obtained: composed by continuous (inner) and digital (external) nested loops. This approach was further developed in [8], where the solvability of the given control problem in the discrete time domain was linked to the exact computation of the sampled representation of the nonlinear model.

The interest of the proposed approach has been confirmed by a large variety of applications: mobile robotics, both for wheeled and legged cases ([11], [15], [13]), aircraft [9], space and underwater ([10], [14]) control problems have been successfully addressed and solved. The possibility of using such a technique in a so large number of cases shows also that the conditions to be fulfilled for its application are not so restrictive.

First results on the existence of a coordinates transformation which renders the sampled representation of nonlinear dynamics finitely computable, as well as conditions of finite computability under a preliminary continuous state feedback, have been set in [8].

P. Di Giamberardino and S. Monaco are with the Dipartimento di Informatica e Sistemistica "Antonio Ruberti", Università di Roma "La Sapienza", Via Eudossiana 18, 00184 Roma, ITALIA, digiamberardino@dis.uniroma1.it, salvatore.monaco@uniroma1.it

D. Normand-Cyrot is with the Laboratoire des Signaux et Systèmes, CNRS-ESE, Plateau de Moulon, 91190 Gif-sur-Yvette, FRANCE, Cyrot@lss.supelec.fr
In this paper it is shown that a driftless dynamics is equivalent, respectively feedback equivalent, to a finitely computable one if and only if the controllability Lie algebra is nilpotent, respectively the control distribution admits a nilpotent basis. It is also shown that, for the class of underactuated mechanical systems, the same conditions must be verified when applied to the kinematic equations.

The presentation is organized as follows: after the statement of the problem and some definitions presented in section II, the equivalence (in section III) and the feedback equivalence (section IV) between driftless dynamics and finitely computable representations are discussed. In section $\mathrm{V}$ the presence of the drift is investigated for the case of underactuated mechanical systems. For each of these cases, necessary and sufficient conditions for the finite computability of the sampled representation under coordinates transformation and state feedback are given.

Unfortunately, in spite of the simple and elegant formulation, well defined procedures which help in checking and computing the desired solution are available only for specific classes of models. For the special class of dynamics admitting the chained form [6], which have been recognized of interest in several real applications, the proposed approach brings to constructive solutions that are given in the paper.

\section{STATEMENT OF THE PROBLEM}

Let us consider a continuous-time system $\Sigma_{c}(f, h)$ described by

$$
\begin{aligned}
\dot{x}_{c}(t) & =f\left(x_{c}(t) ; u(t)\right) \\
y_{c}(t) & =h\left(x_{c}(t)\right)
\end{aligned}
$$

where $x_{c} \in \mathbb{R}^{n}, u \in \mathbb{R}^{m}, y \in \mathbb{R}^{p} . f(\cdot, \cdot)$ and $h(\cdot)$ are analytic in their arguments.

Assuming $u(t)$ constant over time intervals of amplitude $\delta$,

$$
u(k \delta+\tau)=u(k \delta)=u(k) \quad \text { for } 0 \leq \tau<\delta, \quad k \geq 0,
$$

the discrete-time state space representation, $\Sigma_{d}\left(F^{\delta}, h\right)$

$$
\begin{aligned}
x_{d}(k+1) & =F^{\delta}\left(x_{d}(k), u(k)\right) \\
y_{d}(k) & =h\left(x_{d}(k)\right)
\end{aligned}
$$

is the sampled equivalent of $\Sigma_{c}$ if $x_{d}(0)=x_{c}(0)$ implies $x_{d}(k)=x_{c}(k \delta), \forall k>0$.

The sampled dynamics can be computed by integrating the continuous dynamics over the time interval $\delta$ during which the control is constant. 
Otherwise, since the following Taylor series expansion holds true

$$
\begin{aligned}
& x_{c}((k+1) \delta):=x_{c}(k \delta)+\delta\left(\frac{d x_{c}(t)}{d t}\right)_{t=k \delta}+ \\
& +\ldots+\frac{\delta^{p}}{p !}\left(\frac{d^{p} x_{c}(t)}{d t^{p}}\right)_{t=k \delta}+\ldots= \\
& =\left(1+\delta L_{f(\cdot ; u(k))}+\cdots+\frac{\delta^{p}}{p !} L_{f(\cdot ; u(k))}^{p}+\cdots\right)\left(x_{d}(k)\right)
\end{aligned}
$$

whose convergence is assumed for any constant input $u(k)$ and $\delta$ small enough, setting $x_{c}(k \delta)=x_{d}(k), \forall k \geq 0$, a sampled representation is obtained.

$\Sigma_{d}$ in (2) gives an exact sampled representation, ESR, of $\Sigma_{c}$ in (1); it will be called a computable-exact sampled representation, C-ESR, whenever a closed form for $F^{\delta}\left(x_{d}, u\right)$ exists.

A particular case of interest, here investigated, occurs when in (3) the sum into the parenthesis is finite [12], i.e.

$$
\exists P>0: L_{f(\cdot ; u(k))}^{p}\left(x_{d}(k)\right)=0 \quad \forall p>P, \forall u(k) \in \mathbb{R}^{m} .
$$

One obtains in this case a finitely computable - exact sampled representation, FC-ESR, of (1). It is worthy to note that the existence of a FC-ESR is not coordinate free, since a FC-ESR, under a generic diffeomorphism, is transformed into a C-ESR. On the other hand, the existence of a C-ESR is coordinate free.

With this in mind, it is possible to formulate the following problems.

The Finite Computability Equivalence Problem (FCEProblem) is solvable if and only if, in suitable coordinates, the ESR of (1) is finitely computable.

The Finite Computability Feedback Equivalence Problem (FCFE-Problem) is solvable if and only if, under suitable state feedback and coordinates transformation, the ESR of (1) is finitely computable.

\section{EQUIVALENCE BETWEEN DRIFTLESS DYNAMICS AND FC-ESR}

In this section, necessary and sufficient conditions under which a FC-ESR of a certain class of systems can be achieved, are given.

To this aim, it is useful to recall the following result, stated by P. E. Crouch and here reported as Lemma 1.

With reference to (1), denoting by $S$ the Lie algebra generated by the vector fields $f, g_{1}, \ldots, g_{m}$ and $I$ the ideal in $S$ generated by $g_{1}, \ldots, g_{m}$, one has

Lemma 1[1] Given a strongly accessible weakly observable realization, with form as in (1), of a finite Volterra series of length $p, S$ is nilpotent, with a descending central series of length less or equal to $p$, and I is solvable.

A Volterra series associated to a given dynamics (1) corresponds to an integro-differential series expansion with respect to the input $u(t)$. Its expression is given by

$$
\begin{aligned}
& x_{c}((k+1) \delta)= \\
& =H_{0}(\cdot)+\sum_{i=1}^{m} H_{1}^{i}(\cdot) u_{i}(k)+\ldots+ \\
& +\sum_{i_{1}, \ldots, i_{p}=1}^{m} H_{p}^{i_{1}, \ldots, i_{p}}(\cdot) u_{i_{1}}(k) \ldots u_{i_{p}}(k)+\ldots
\end{aligned}
$$

where

$$
\begin{aligned}
H_{0}(\cdot) & =H_{0}\left(t, k \delta ; x_{c}(k \delta)\right) \\
H_{1}^{i}(\cdot) & =\int_{k \delta}^{t} H_{1}^{i}\left(t, \sigma_{1} ; x_{c}(k \delta)\right) d \sigma_{1} \\
\vdots & \\
H_{p}^{i_{1}, \ldots, i_{p}}(\cdot) & =
\end{aligned}
$$$$
=\int_{k \delta}^{t} \ldots \int_{k \delta}^{\sigma_{(p-1)}} H_{p}^{i_{1}, \ldots, i_{p}}\left(t, \sigma_{1}, \ldots, \sigma_{p} ; x_{c}(k \delta)\right) d \sigma_{1} \ldots d \sigma_{p}
$$

evaluated for $t=(k+1) \delta$.

From now on it will be assumed that in (1)

$$
f\left(x_{c}(t) ; u(t)\right)=\sum_{i=1}^{m} u_{i}(t) g_{i}\left(x_{c}(t)\right)
$$

where the vector fields $g_{i}, i=1, \ldots, m$, are such that, on an open $\mathcal{M} \subseteq \mathbb{R}^{n}$ :

(i. ) the distribution $\mathcal{D}=\operatorname{span}\left\{g_{1}, \ldots, g_{m}\right\}$ has dimension $m$;

(ii.) $\mathcal{L}=\operatorname{Lie}\left\{g_{1}, \ldots, g_{m}\right\}$, the Lie algebra they generate, is full rank.

Remark: (i. ) states the independency of the control action, while (ii.) corresponds to assume the full controllability of (7) $[4]$.

It is now possible to give the following result.

Theorem 1 The finite computability equivalence problem is solvable for the driftless dynamics (7) if and only if $\mathcal{L}$ is nilpotent.

Proof The sufficiency has been proved in [8], to which the reader is referred, making use of the concept of dilations [2] induced by the nilpotent Lie algebra generated by the vector fields $g_{1}, \ldots, g_{m}$.

As far as the necessity is concerned, the first step is based on the verification that the finite computability of (7), i.e. the fulfillment of condition (4), implies the finiteness of the Volterra series associated with the input-state representation computed at time $t=(k+1) \delta$ starting from $t_{0}=k \delta$ with $x_{c}\left(t_{0}\right)=x_{c}(k \delta)$, so proving that finite computable driftless dynamics generate finite Volterra series. 
Since for the case under study, with reference to (5) and (6), one has

$$
\begin{aligned}
H_{0}(\cdot) & =x_{c}(k \delta) \\
H_{1}^{i}(\cdot) & =L_{g_{i}} H_{0}(\cdot)=L_{g_{i}} x_{c}(k \delta) \\
\vdots & \vdots \\
H_{p}^{i_{1}, \ldots, i_{p}}(\cdot)= & L_{g_{i_{p}}} H_{(p-1)}^{i_{1}, \ldots, i_{(p-1)}}(\cdot)= \\
= & L_{g_{i_{p}}} L_{g_{i_{(p-1)}}} \cdots L_{g_{i_{1}}} x_{c}(k \delta)
\end{aligned}
$$

then (5) can be rewritten as

$$
\begin{aligned}
& x_{c}((k+1) \delta)= \\
& =x_{c}(k \delta)+\int_{k \delta}^{(k+1) \delta} d \sigma_{1} \Sigma_{1}+\ldots+ \\
& \quad+\int_{k \delta}^{(k+1) \delta} \cdots \int_{k \delta}^{\sigma_{(p-1)}} d \sigma_{1} \ldots d \sigma_{p} \Sigma_{p}+\ldots
\end{aligned}
$$

where

$$
\begin{aligned}
\Sigma_{1} & =\sum_{i=1}^{m} L_{g_{i}} x_{c}(k \delta) u_{i}(k) \\
\vdots & \vdots \\
\Sigma_{p} & =\sum_{i_{1}, \ldots, i_{p}=1}^{m} L_{g_{i_{p}}} \ldots L_{g_{i_{1}}} x_{c}(k \delta) u_{i_{1}}(k) \ldots u_{i_{p}}(k)
\end{aligned}
$$

From the identity

$$
\begin{gathered}
L_{\sum_{i=1}^{m} u_{i}(k) g_{i}(\cdot)}^{p}\left(x_{c}(k \delta)\right)= \\
=\sum_{i_{1}, \ldots, i_{p}=1}^{m} L_{g_{i_{1}}} \ldots L_{g_{i_{p}}} x_{c}(k \delta) u_{i_{1}}(k) \ldots u_{i_{p}}(k)
\end{gathered}
$$

it is clear that (4) is equivalent to $H_{p}^{i_{1}, \ldots, i_{p}}(\cdot)=0$ for $p>P$, i.e. the finiteness of (8), being $\Sigma_{p}=0$ for $p>P$.

Now, making use of Lemma 1 under the assumption (7), meaning that the Lie algebra $S$ and the ideal $I$ becomes coincident, finiteness of the Volterra series implies the nilpotency of the Lie algebra $\mathcal{L}$.

Remark: The practical application of the previous result requires to test the nilpotency of $\mathcal{L}$ and to compute the (local) diffeomorphism defining the coordinates change.

The first step is quite easy to be performed, since one has to verify that all the Lie products (brackets) between vector fields $g_{i}$ of order greater than a certain value $P$ are equal to zero.

Alternatively, a different approach can result easier to be followed if some software tool for symbolic computation, as Mathematica or Maple for instance, is used. In fact, starting from the Lie algebra $\mathcal{L}$, one can introduce its central descending series recursively defined as ${ }^{1}$

$$
\begin{gathered}
\mathcal{L}^{1}=\mathcal{L} \\
\mathcal{L}^{k+1}=\left[\mathcal{L}, \mathcal{L}^{k}\right]
\end{gathered}
$$

The nilpotency of $\mathcal{L}$ can be verified checking the existence of an integer $P$ such that $\mathcal{L}^{p}=\{0\}$ for $p>P$.

\footnotetext{
${ }^{1}$ According to the notation used in [2]
}

A. How to compute the coordinates change $z=\Phi(x)$

From [2] we recall in the sequel how to compute the coordinates transformation.

Step 1: Compute the central descending series of $\mathcal{L}$;

Step 2: compute the $n$ integers $r_{i}, i=1, \ldots, n$, such that

$$
r_{i}=\max \left\{j \in Z^{+}: \operatorname{dim} \mathcal{L}^{j}(\bar{x}) \geq n+1-i\right\}, \quad x \in \mathcal{M}
$$

Step 3: choose $Y^{1}, \ldots, Y^{n} \in \mathcal{L}$ such that $Y^{i} \in \mathcal{L}^{r_{i}}, i=$ $1, \ldots, n$, with $Y^{1}(\bar{x}), \ldots, Y^{n}(\bar{x})$ linearly independent;

Step 4: compute the map

$$
x=\gamma(z)=\left(\exp z_{n} Y^{n}\right) \cdots\left(\exp z_{1} Y^{1}\right)(\bar{x})
$$

Step 5: compute $z=\Phi(x)=\gamma^{-1}(x)$.

Example Let a two inputs three dimensional driftless dynamics be described by the two vector fields

$$
\begin{aligned}
g_{1}= & \cos \left(x_{3}\right) \frac{\partial}{\partial x_{1}}+\sin \left(x_{3}\right) \frac{\partial}{\partial x_{2}} \\
g_{2}= & \left(x_{1} \sin \left(x_{3}\right) \cos \left(x_{3}\right)-x_{2} \cos ^{2}\left(x_{3}\right)\right) \frac{\partial}{\partial x_{1}}+ \\
& +\left(x_{1} \sin ^{2}\left(x_{3}\right)-x_{2} \sin \left(x_{3}\right) \cos \left(x_{3}\right)\right) \frac{\partial}{\partial x_{2}}+\frac{\partial}{\partial x_{3}}
\end{aligned}
$$

that, as it is easily verified, generate a nilpotent Lie algebra, since the only nonzero Lie bracket is

$$
\left[g_{1}, g_{2}\right]=\sin \left(x_{3}\right) \frac{\partial}{\partial x_{1}}-\cos \left(x_{3}\right) \frac{\partial}{\partial x_{2}} .
$$

The application of the previous algorithm gives

$$
\begin{gathered}
\mathcal{L}^{1}=\left\{g_{1}, g_{2},\left[g_{1}, g_{2}\right]\right\}, \quad \mathcal{L}^{2}=\left\{\left[g_{1}, g_{2}\right]\right\}, \quad P=2 \\
r_{1}=1, \quad r_{2}=1 \quad \text { and } \quad r_{3}=2 \\
Y^{1}=\frac{\partial}{\partial x_{3}}, \quad Y^{2}=g_{1} \text { and } Y^{3}=\left[g_{1}, g_{2}\right] \\
\gamma(z)=\left(\begin{array}{c}
z_{2} \cos \left(z_{1}\right)+z_{3} \sin \left(z_{1}\right) \\
z_{2} \sin \left(z_{1}\right)-z_{3} \cos \left(z_{1}\right) \\
z_{1}
\end{array}\right) \\
x_{3} \\
\Phi(x)=\left(\begin{array}{c}
x_{1} \cos \left(x_{3}\right)+x_{2} \sin \left(x_{3}\right) \\
x_{1} \sin \left(x_{3}\right)-x_{2} \cos \left(x_{3}\right)
\end{array}\right)
\end{gathered}
$$

Once applied, the so obtained coordinates change transforms $g_{1}$ and $g_{2}$ into

$$
\tilde{g}_{1}=\frac{\partial}{\partial z_{2}}, \quad \tilde{g}_{2}=\frac{\partial}{\partial z_{1}}+z_{2} \frac{\partial}{\partial z_{3}} .
$$

\section{FEEDBACK EQUIVALENCE BETWEEN DRIFTLESS DYNAMICS AND FC-ESR}

The possibility of dealing with systems whose dynamics fulfill the nilpotency property is very rare: this fact strongly limits the applicability of the previous result. The possibility of rendering the ESR of a given plant finitely computable modifying it by a continuous static state feedback has been proposed as a powerful tool in the design of discontinuous feedback control laws ([8], [13]). 
In view of Theorem 1 , the problem reduces to the existence and the computation of a feedback under which the system is nilpotent.

Starting from the basic result by Hermes [3] and the ones in [8], the following Theorem can be stated.

Theorem 2 The finite computability feedback equivalence problem is solvable for the driftless dynamics (7) if and only if $\mathcal{D}$ admits a nilpotent basis.

Proof Starting from (1) under assumption (7)

$$
\dot{x}=\sum_{i=1}^{m} u_{i} g_{i}(x)
$$

one can get

$$
\sum_{j=1}^{m} v_{j} \tilde{g}_{j}(x)
$$

with $\operatorname{Lie}\left(\tilde{g}_{1}, \ldots, \tilde{g}_{m}\right)$ nilpotent, under an invertible state feedback

$$
u=\beta(x) v
$$

if and only if it is possible to write

$$
g_{i}=\sum_{j=1}^{m} \alpha_{i, j}(x) \tilde{g}_{j} \quad i=1, \ldots, m
$$

with

$$
\left(\begin{array}{ccc}
\alpha_{1,1}(x) & \ldots & \alpha_{m, 1}(x) \\
\vdots & \ddots & \vdots \\
\alpha_{1, m}(x) & \ldots & \alpha_{m, m}(x)
\end{array}\right)^{-1}=\beta(x)
$$

In fact, in this case

$$
\begin{aligned}
\dot{x}= & \sum_{i=1}^{m} u_{i} g_{i}=\sum_{i=1}^{m} u_{i} \sum_{j=1}^{m} \alpha_{i, j}(x) \tilde{g}_{j}= \\
& \sum_{j=1}^{m}\left(\sum_{i=1}^{m} u_{i} \alpha_{i, j}(x)\right) \tilde{g}_{j}=\sum_{j=1}^{m} v_{j} \tilde{g}_{j} .
\end{aligned}
$$

Since (9) means that $\tilde{g}_{1}, \ldots, \tilde{g}_{m}$ is a (nilpotent) basis for $\mathcal{D}$, and Theorem 1 states that nilpotency is a necessary and sufficient condition for equivalence to finitely computable dynamics, the theorem is proved.

Remark Unfortunately, no results to check the existence of a nilpotent basis of a given distribution are available. At authors knowledge, the only available constructive results on this subject are the ones in [3]. In the sequel, they are shortly recalled.

\section{Procedure 1}

Step 1 Set $\mathcal{G}=\left\{g_{1}, \ldots, g_{m}\right\}$ the given initial basis of the distribution $\mathcal{D}$;

Step 2 compute $\mathcal{G}^{i}$ as the sets of $i$-tuples of $g_{1}, \ldots, g_{m}$, $i=1,2, \ldots$;

Step 3 choose any nilpotent basis $\mathcal{X}=\left\{X^{1}, \ldots, X^{m}\right\}$ for a distribution on $\mathbb{R}^{n}$, such that, once the sets of $i$-tuples $\mathcal{X}^{i}(\xi)$ of $X^{1}, \ldots, X^{m}$ have been computed, dim span $\mathcal{X}^{i}(\xi)=\operatorname{dim}$ span $\mathcal{G}^{i}(x), i=1,2, \ldots$ (the necessity of such a condition can be found in [3]);

Step 4 find a diffeomorphism $\phi: \mathbb{R}^{n} \rightarrow M$ such that $\phi_{*} X^{1}, \ldots, \phi_{*} X^{m}$ is a basis for $\mathcal{D}$. The solution, when it exists, can be obtained solving a system of first order partial differential equations with respect to the unknown diffeomorphism $\phi$.

\section{Procedure 2}

It applies when $m=n-1$ and makes use of the Darboux theorem [16] here recalled: if a 1-form $\omega$ has constant rank $r$ in a neighborhood of $\bar{x}$, then there exist local coordinates $x_{1}, \ldots, x_{n}$ such that

$$
\omega=d x_{1}+x_{2} d x_{3}+\ldots+x_{2 r} d x_{2 r+1} .
$$

Then, one has

Step 1 compute the 1 -form $\omega$ such that $\left\langle\omega(x), g_{i}(x)\right\rangle=0$, $i=1, \ldots, n-1$, for $x$ in a neighborhood of $\bar{x} \in \mathcal{M}$ and check if it has constant rank $r \geq 1$ in the same neighborhood. If it does, go to step 2, otherwise this procedure fails.

Step 2 By Darboux theorem, a nilpotent basis for $\mathcal{D}$ is given by

$$
\tilde{g}_{1}=-x_{2} \frac{\partial}{\partial x_{1}}+\frac{\partial}{\partial x_{3}}
$$

$$
\begin{gathered}
\tilde{g}_{r}=-x_{2 r} \frac{\partial}{\partial x_{1}}+\frac{\partial}{\partial x_{2 r+1}} \\
\tilde{g}_{r+1}=\frac{\partial}{\partial x_{2}}, \quad \tilde{g}_{r+2}=\frac{\partial}{\partial x_{4}}, \cdots \quad \tilde{g}_{2 r}=\frac{\partial}{\partial x_{2 r}} \\
\tilde{g}_{2 r+1}=\frac{\partial}{\partial x_{2 r+2}}, \quad \tilde{g}_{2 r+2}=\frac{\partial}{\partial x_{2 r+3}}, \cdots \tilde{g}_{n-1}=\frac{\partial}{\partial x_{n}}
\end{gathered}
$$

since they satisfy $<\omega(x), \tilde{g}_{i}(x)>=0, i=1, \ldots, n-1$, and nilpotency is easily verified.

In this case, relationships with chained forms [6] are easily established.

Example Let the two inputs three dimensional driftless dynamics be described by

$$
g_{1}=\cos \left(\xi_{3}\right) \frac{\partial}{\partial \xi_{1}}+\sin \left(\xi_{3}\right) \frac{\partial}{\partial \xi_{3}} \quad g_{2}=\frac{\partial}{\partial \xi_{3}} .
$$

A 1 -form $\omega$ such that $\left\langle\omega, g_{i}\right\rangle(\xi)=0$ is given by

$$
\omega=\sin \left(\xi_{3}\right) d \xi_{1}-\cos \left(\xi_{3}\right) d \xi_{2} .
$$

Under the coordinates change

$$
x=\left(\begin{array}{c}
\xi_{1} \sin \left(\xi_{3}\right)-\xi_{2} \cos \left(\xi_{3}\right) \\
-\xi_{1} \cos \left(\xi_{3}\right)-\xi_{2} \sin \left(\xi_{3}\right) \\
\xi_{1}
\end{array}\right)
$$

(11) assumes the form (10), as it can be verified by simple computations. Then, the nilpotent basis w.r.t. the new coordinates is easily obtained. Reverse computations give for the nilpotent bases w.r.t. the original coordinates

$$
\begin{aligned}
\tilde{g}_{1}= & \left(\xi_{1} \sin \left(\xi_{3}\right) \cos \left(\xi_{3}\right)-\xi_{2} \cos ^{2}\left(\xi_{3}\right)\right) \frac{\partial}{\partial \xi_{1}}+ \\
& +\left(\xi_{1} \sin ^{2}\left(\xi_{3}\right)-\xi_{2} \sin \left(\xi_{3}\right) \cos \left(\xi_{3}\right)\right) \frac{\partial}{\partial \xi_{2}}+\frac{\partial}{\partial \xi_{3}} \\
\tilde{g}_{2}= & -\cos \left(\xi_{3}\right) \frac{\partial}{\partial \xi_{1}}-\sin \left(\xi_{3}\right) \frac{\partial}{\partial \xi_{2}}
\end{aligned}
$$




\section{UNDERACTUATED MECHANICAL SYSTEMS}

In this section the previous result on driftless dynamics is extended to the class of underactuated mechanical systems. Such a class of systems is characterized by a mathematical model, obtained from a classical Euler-Lagrange approach, that can be written in the form [5]

$$
J(q) \ddot{q}=F(q, \dot{q})+G(q)+\omega^{T}(q) \lambda+B(q, \dot{q}) \tau
$$

with $q \in \mathbb{R}^{n}$ is the generalized coordinates vector, $\tau \in \mathbb{R}^{(n-m)}$ is the input vector, $\lambda \in \mathbb{R}^{m}$ and $J(\cdot), F(\cdot, \cdot), G(\cdot), \omega(\cdot)$ and $B(\cdot, \cdot)$ are full rank matrices with real analytic entries.

In (12), $\omega^{T}(x)$ denotes the $m$ (local) directions along which the controls $\tau_{i}$ do not act. It verifies $\omega(q) \dot{q}=0$, a kinematic constraint that, being not integrable, makes the dynamics (12) a nonholonomic one.

In this case, a drift is present in the dynamics and the results given in the previous sections, being referred to driftless systems, do not apply.

However, in [10] the authors have shown that dynamics (12) is feedback equivalent to

$$
\begin{aligned}
\dot{x} & =K(x) u \\
\dot{u} & =a
\end{aligned}
$$

denoting it as a LADIC (Linear Analytic and Driftless under Integral Control). More precisely, the structure (13) can be obtained, after state feedback and coordinates change, when a system described by the classical Euler-Lagrange equations is characterized by nonintegrable kinematic constraints or, in other words, nonholonomic constraints.

It is useful to recall from [10] the definition of $K(x)$. Let $\gamma_{1}(q), \ldots, \gamma_{m}(q)$ be a basis for the subspace orthogonal to $\omega(q) J^{-1}(q)$. Setting $\Gamma(q)$ as

$$
\Gamma(q)=\left(\begin{array}{lll}
\gamma_{1}(q) & \cdots & \gamma_{m}(q)
\end{array}\right)
$$

it satisfies, by definition,

$$
\omega(q) J^{-1}(q) \Gamma(q)=0 .
$$

Consider, finally, the nonsingular (see [10]) matrix

$$
H(q)=\left(\begin{array}{c}
\Gamma^{T}(q) \\
\omega(q)
\end{array}\right)
$$

Then, $K(x)$ is obtained as the first $m$ columns of $H(q)^{-1}$ once that the "coordinates change" $x=q$ is performed. This means that the following relationships must hold

$$
\begin{aligned}
\Gamma^{T}(q) K(q) & =\Gamma^{T}(x) K(x)=I \\
\omega(q) K(q) & =\omega(x) K(x)=0
\end{aligned}
$$

Now the results presented in the previous sections can be used for giving conditions about the finite computability equivalence and feedback equivalence problems for LADIC systems and, thanks to the relationships between (13) and (12), also for general systems as (12).
In fact, in [10] it has been proved that a LADIC dynamics is equivalent (feedback equivalent) to a FC-ESR if and only if its kinematic part

$$
\dot{x}=K(x) u=\sum_{i=1}^{m} k_{i}(x) u_{i}
$$

satisfies the same property. Then, making use of the results given in sections III and IV, one has

Proposition 1 The finite computability equivalence problem (finite computability feedback equivalence problem) is solvable for the LADIC dynamics (13) if and only if the vector fields $k_{i}$ of its kinematic part are nilpotent (admit a nilpotent basis).

Then, it is possible to state conditions directly on the terms appearing in (12).

First of all, comparing (14) and (16) and taking into account the full rank condition on the involved matrices, it is possible to write for $K(x)$ also the expression

$$
K(x)=J^{-1}(x) \Gamma(x) T(x)
$$

with $T(x)$ any nonsingular $m \times m$ matrix. Using (17) in (15) one gets

$$
\Gamma^{T}(x) J^{-1}(x) \Gamma(x) T(x)=I
$$

satisfied only for $T(x)=I$, due to the positive definiteness of $J^{-1}(x)^{2}$

It is possible to conclude for the finite computability equivalence problem that

Proposition 2 A LADIC form obtained from a dynamics as in (12) admits a FC-ESR if and only if the $m$ vector fields $J^{-1}(x) \gamma_{1}(x), \ldots, J^{-1}(x) \gamma_{m}(x)$ generate a nilpotent Lie algebra.

As far as the finite computability feedback equivalence problem is concerned, a possible state feedback on the kinematic part of (13) must be considered. In fact, as well known, a state feedback

$$
u_{i}=\sum_{h=1}^{m} r_{i, h}(x) v_{h}
$$

corresponds to the change of basis for the distribution $K=$ $\operatorname{span}\left\{k_{1}, \ldots, k_{m}\right\}$.

$$
\begin{aligned}
\dot{x} & =\sum_{i=1}^{m} k_{i}(x) u_{i}=\sum_{i=1}^{m} k_{i}(x) \sum_{h=1}^{m} r_{i, h}(x) v_{h}= \\
& =\sum_{h=1}^{m} \sum_{i=1}^{m} k_{i}(x) r_{i, h}(x) v_{h}=\sum_{h=1}^{m} \tilde{k}_{h}(x) v_{h}
\end{aligned}
$$

with

$$
\tilde{k}_{h}(x)=\sum_{i=1}^{m} k_{i}(x) r_{i, h}(x)
$$

or, in a compact matrix form

$$
\tilde{K}(x)=K(x) R(x)
$$

\footnotetext{
${ }^{2}$ From $x^{T} J x>0$, true $\forall x \neq 0$ by definition of the symmetric inertia matrix $J$, choosing $x=J^{-1} v$ one has $v^{T} J^{-T} J J^{-1} v=v^{T} J^{-T} v=$
$v^{T} J^{-1} v>0$.
} 
with $R(x)$ not singular and such that each term $r_{i, h}$ represents its entry $(h, i)$.

Such a transformation is compatible with condition (18) once one identifies $T(x)$ and $R(x)$. As far as (15) is concerned, one has

$$
\Gamma^{T}(x) \tilde{K}(x)=\Gamma^{T}(x) K(x) R(x)=R(x)
$$

that implies, in order to maintain such a condition, that $\Gamma(x)$ must change. This change is defined as

$$
\tilde{\Gamma}(x)=\Gamma(x) R^{-T}(x)
$$

so that

$$
\tilde{\Gamma}^{T}(x) \tilde{K}(x)=I
$$

From (14)

$$
\omega(x) J^{-1}(x) \tilde{\Gamma}(x)=\omega(x) J^{-1}(x) \Gamma(x) R^{-T}(x)=0
$$

so that, regarding the expression

$$
J^{-1}(x) \Gamma(x) R^{-T}(x)
$$

as a basis change for the distribution

$$
\Delta=\operatorname{span}\left\{J^{-1}(x) \gamma_{1}(x), \ldots, J^{-1}(x) \gamma_{m}(x)\right\}
$$

from Proposition 2 one has

Proposition 3 The finite computability feedback equivalence problem for a LADIC form obtained from a dynamics as in (12) has a solution if and only if the $m$ vector fields $J^{-1}(x) \gamma_{1}(x), \ldots, J^{-1}(x) \gamma_{m}(x)$ admits a basis that generates a nilpotent Lie algebra.

From Propositions 2 and 3, the only difference between the conditions for finite computability equivalence problem and finite computability feedback equivalence problem for a LADIC form is in the fulfillment of the nilpotent condition on the vector fields $J^{-1}(x) \gamma_{i}(x)$ directly or under change of basis. This fact, in conjunction with the freedom in choosing the vector fields $\gamma_{i}(x)$ for the transformation into LADIC form, brings to the conclusion

Proposition 4 An underactuated (nonholonomic) dynamical system as (12) is feedback equivalent to a LADIC form for which the FCE-Problem admits a solution if and only if it is feedback equivalent to a LADIC form for which the FCDEProblem does.

\section{CONCLUSIONS}

In this paper the conditions under which a driftless dynamics is equivalent or feedback equivalent to a finitely computable one, i.e. a dynamics for which it is possible to compute a FC-ESR, have been studied. It has been proved that nilpotency of the Lie algebra $\mathcal{L}$ generated by the vector fields $g_{i}$ that characterize the dynamics is a necessary and sufficient condition for equivalence while the existence of a nilpotent basis for the distribution $\mathcal{D}$ is a necessary and sufficient condition for the feedback equivalence. For the class of underactuated mechanical systems it has been shown that the same conditions must be verified on the kinematic equations.

The importance of driftless dynamics as well as the underactuated ones in many applicative fields motivates the interest of the results.

Two major open problems, at present under study, need to be further investigated: the settlement of algorithms for checking the existence of a nilpotent basis of a given distribution and the study of more general dynamics with drift.

\section{REFERENCES}

[1] Crouch P. E.: Dynamical Realizations of Finte Volterra Series, SIAM J. of Control and Optimization, 1981, 19, 2, 177-202

[2] Kawski M.: Nilpotent Lie Algebras of Vector Fields, Journ. für die reine und angewandte Mathematik, 1988, 388, 1-17.

[3] Hermes H., Lundell A. and Sullivan D.: Nilpotent Bases for Distributions and Control Systems, J. of Differential Equations, 1984, 55, $385-400$

[4] Sussmann H. J.: A general theorem on local controllability, SIAM J. Control and Optimization, 1987, 25, 1, 158-194.

[5] Kolmanowsky, I. and McClamroch, N.H., Developments in Nonholonomic Control Problems, IEEE Control Systems Magazines, 1995, 15, n.6, 20-36

[6] Murray R. M., Sastry S. S.: Nonholonomic Motion Planning: Steering Using Sinusoids, IEEE Trans. Aut. Contr., 1993, 38, 5.

[7] Monaco S., Normand-Cyrot D.: An Introduction to Motion Planning under Multirate Digital Control, in Proc. 31st IEEE Control and Decision Conference, 1992, 1780-1785.

[8] Di Giamberardino P., Monaco S. and Normand-Cyrot D.: Digital control through finite feedback discretizability, Proc. Int. Conference on Robotics and Automation, 1996, 3141-3146.

[9] Di Giamberardino P., Djemai M., Monaco S. and Normand-Cyrot D.: Exact Steering and Stabilization of a PVTOL Aircraft, in Proc. 36th IEEE Control and Decision Conference, 1997, 2049-2054.

[10] Di Giamberardino P., Monaco S. and Normand-Cyrot D.: A Hybrid Control Scheme for Maneuvering Space Multibody Systems, J. of Guidance, Control and Dynamics, 2000, 23, n.2, 231-240.

[11] Di Giamberardino P., La Gala F., Monaco S. and Normand-Cyrot D.: Experimental Torque Control of a Wheeled Robot under Digital Motion Planning, in Proc. 20th IASTED Int. Conf. on Modelling, Identification and Control, 2001.

[12] Monaco S. and Normand-Cyrot D.: Issues on nonlinear digital control, European Journal of Control, 2001, 7, 2-3. 160-178.

[13] Di Giamberardino P., Monaco S. and Normand-Cyrot D.: Why Multirate Sampling is Instrumental for Control Design Purpose: the example of the one-leg hopping robot, in Proc. 41st IEEE Control and Decision Conference, 2002.

[14] Di Giamberardino P. and Sabbadini F.: Control of an Underactuated Underwater Robotic Manipulator, in Proc. 11th IEEE Mediterranean Conference on Control and Automation, 2003.

[15] Usai A. and Di Giamberardino P.: Nonholonomic mobile robot visual servoing: a multirate digital approach, in Proceedings 8th WSEAS Int. Conf. on Automatic Control, Modeling and Simulation, pp. 134-139, 2006.

[16] Bryant R. L., Chern S. S., Gardner R. B., Goldschmidt H. L. and Griffiths P. A.: Exterior Differential Systems, Mathematical Sciences Research Institute Publications, 18, Springer-Verlag, New York, 1991. 